\title{
Intramedullary spinal cord cavernous angioma in children: case report and literature review
}

\author{
Rémy Noudel • Fabien Litré • Mathieu Vinchon • \\ Martine Patey $\cdot$ Pascal Rousseaux
}

Received: 18 May 2007 /Revised: 28 May 2007 /Published online: 11 September 2007

(C) Springer-Verlag 2007

\begin{abstract}
Introduction Intramedullary cavernous angiomas (ImCA) of the spinal cord are very uncommon malformations especially in the pediatric population where only ten cases have yet been reported within the available literature.

Case report In this paper, the authors report the case of a 12year-old girl presenting with a T11 level hematomyelia because of a cavernous angioma. Microsurgical excision was performed with good clinical outcome and no magnetic resonance imaging evidence of residual cavernoma 30 months later.

Discussion Despite the small number of cases reported in children, this one contributes to the literature identifying special features of presentation and management of ImCA for pediatric patients. Because a higher risk of recurrent bleeding has been demonstrated for ImCA, with dramatic clinical consequences, microsurgical removal remains the only definitive treatment.
\end{abstract}

R. Noudel $(\bowtie) \cdot$ F. Litré $\cdot$ P. Rousseaux

Department of Neurosurgery, Maison Blanche Hospital, University of Reims,

Reims, France

e-mail: remynoudel@yahoo.fr

M. Vinchon

Department of Pediatric Neurosurgery, Roger Salengro Hospital, University of Lille,

Lille, France

M. Patey

Department of Neuropathology, University of Reims,

Reims, France
Keywords Cavernoma $\cdot$ Intramedullary cavernous angioma $\cdot$ Hematomyelia $\cdot$ Pediatric Neurosurgery

\section{Introduction}

Cavernous angiomas (CAs) are developmental vascular hamartomas that occur throughout the body, including the central nervous system.

The universal availability of magnetic resonance imaging (MRI) has afforded easy diagnosis of CA, especially in the brain where they account for $1 \%$ of all vascular lesions and $15 \%$ of vascular malformations [5, 17]. Intramedullary spinal cord cavernous malformations are much less common: To our knowledge, only 122 cases have been published until now $[1,3,4,8,11,12,15,17,19]$, with only ten of those in pediatric patients $[1,3,6,14,17]$. This scarcity makes it very difficult to draw some guidelines for the management of these lesions in children.

Microsurgical resection is the only available therapeutic option for intramedullary cavernous malformations [3, 14, 19]. However, indications for operating on asymptomatic cavernous malformations remain controversial; although some authors $[1,12]$ do not recommend surgery in these cases, one has to weigh the risks of surgery against those of sudden hemorrhage with serious neurological consequences in case of surgical abstention. The facts that all cavernous hemangiomas of the central nervous system show histological evidence of previous hemorrhage $[1,18]$ and that catastrophic neurological deterioration caused by hematomyelia has been documented $[1,3,4,14]$ underscore the risks of surgical abstention. 
We report here a case of symptomatic intramedullary spinal cord CAs (ImCA) in a 12-year-old girl treated by complete microsurgical excision. We discuss clinical features and principles of management of these lesions in children in the light of a review of the available literature.

\section{Case report}

\section{History and examination}

This 12-year-old girl presented with an acute but incomplete left T12 Brown-Sequard syndrome. It had started 2 days earlier by low back pain irradiating to the lower limbs. At admission, she exhibited motor weakness involving all muscle groups of the lower limbs but predominating in the distal part causing foot drop. Neurological examination found left Babinski's sign and painful dysesthesia of her right leg with decreased pain and temperature sensation below the level of T12.

MRI revealed an intramedullary ovoid lesion $2 \mathrm{~cm}$ in diameter, located in the left lateral column of the spinal cord at the T11 level. Its signal was mixed in the T2weighted image (Figs. 1 and 2). In the T1-weighted image, it was hyperintense with a peripheral rim of edema. No other lesion was identified along the entire neuraxis. Spinal angiography was negative: It showed that the anterior spinal vascularization was supplied by the left $\mathrm{T} 9$ and right L1 segmental arteries.

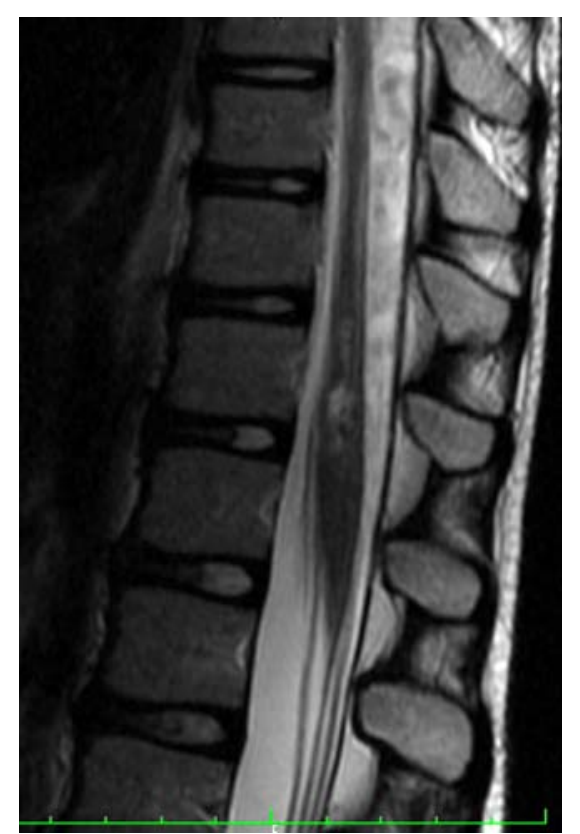

Fig. 1 Preoperative MRI study. The sagittal T2-weighted image shows spontaneous hypersignal of hematomyelia at T11 with a curved image of low signal centrally because of capillary vessels

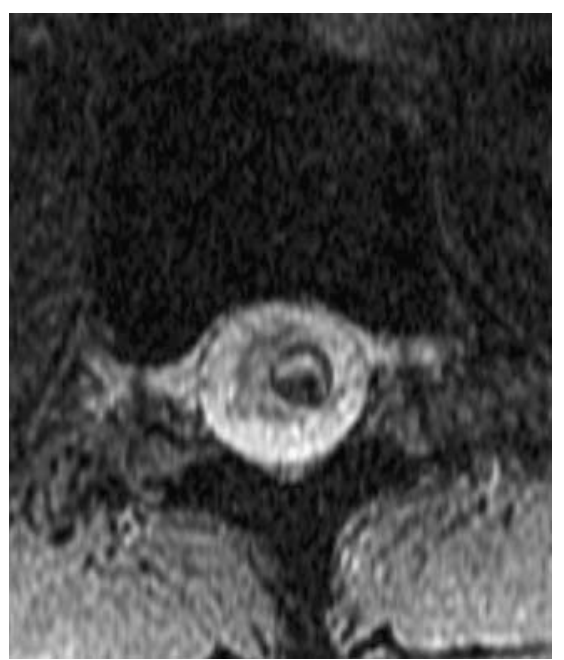

Fig. 2 The preoperative axial T2-weighted image shows an ovoid hypointense lesion located in the left lateral column of the spinal cord with a mixed signal core because of hemorrhage and capillary vessels

Operation and postoperative course

The patient was operated on 4 months after the diagnosis. A T10 to T12 laminotomy was performed using a high-speed craniotome with a pediatric footplate. After opening the dura and arachnoid, no pial anomaly was immediately evident because the CA was located laterally. After the dentate ligament was divided, gentle rotation of the spinal cord to the right allowed us to expose a small irregular area of bluish discoloration at the surface of the lateral column of the spinal cord (Fig. 3). Under magnification with the operative microscope, a limited myelotomy was made directly over this area: A cavernous malformation with the typical mulberry aspect was found just underneath the surface. Dissection was carried along the thin plane of gliotic, hemosiderin-stained tissue surrounding the malformation; this plane was respected to prevent neurological deterioration (Fig. 4). Total resection of the malformation was performed. After watertight dural closure, the T10 to T12 laminae were replaced and sutured using $2-0$ braided nylon sutures.

Histopathological examination of the specimen revealed large vessels with thick fibrous walls and no intervening neural element between the vascular channels (Figs. 5 and 6). Hemosiderin-containing macrophages were present in the normal adjacent spinal cord tissue.

Postoperatively, the patient experienced increased weakness and sensory loss in her left leg, which subsided completely within 3 months. Eighteen months after surgery, neurological examination found only residual left leg pyramidal syndrome without motor weakness; MRI showed a residual postoperative cavity of cerebrospinal fluid intensity without residual cavernoma (Figs. 7 and 8). She wore a brace 


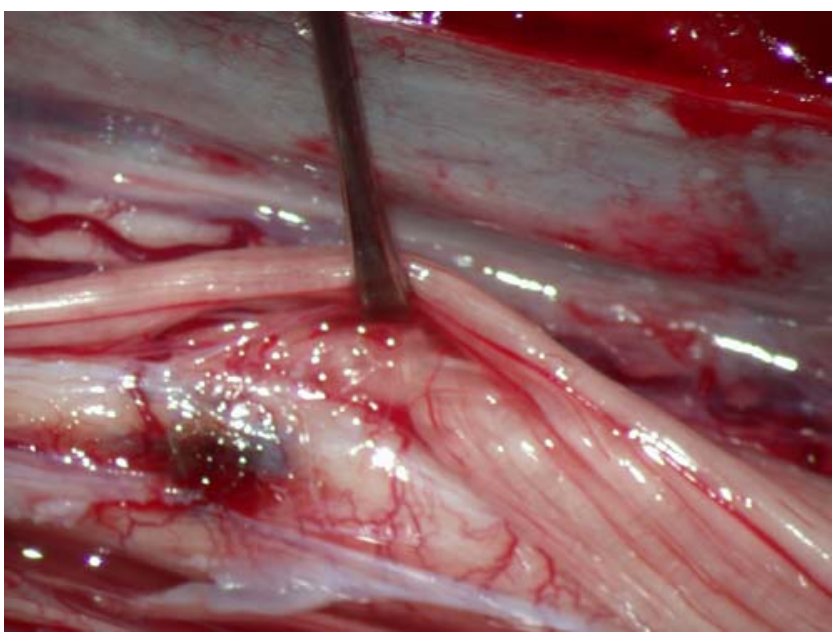

Fig. 3 Intraoperative photograph showing the bluish discoloration at the surface of the left lateral column of the spinal cord from the underlying cavernous angioma

during the 6 months after surgery to prevent spinal deformity that did not occur until now.

\section{Discussion}

CAs are ubiquitous vascular malformations that can affect every organ system throughout the body. Histopathology, immunohistochemistry, and electron microscopy show that all lesions are identical regardless of location [12]. Whereas spinal osseous cavernous malformations are highly vascular lesions with a high risk of massive intraoperative blood loss $[9,12]$, intramedullary and intracerebral CAs are angiographically occult $[1,2,8,12,13,17]$ and can be resected with negligible blood loss.

MRI is the gold standard for diagnosis in any location and typically shows a small, round- or ovoid-shaped, well-defined

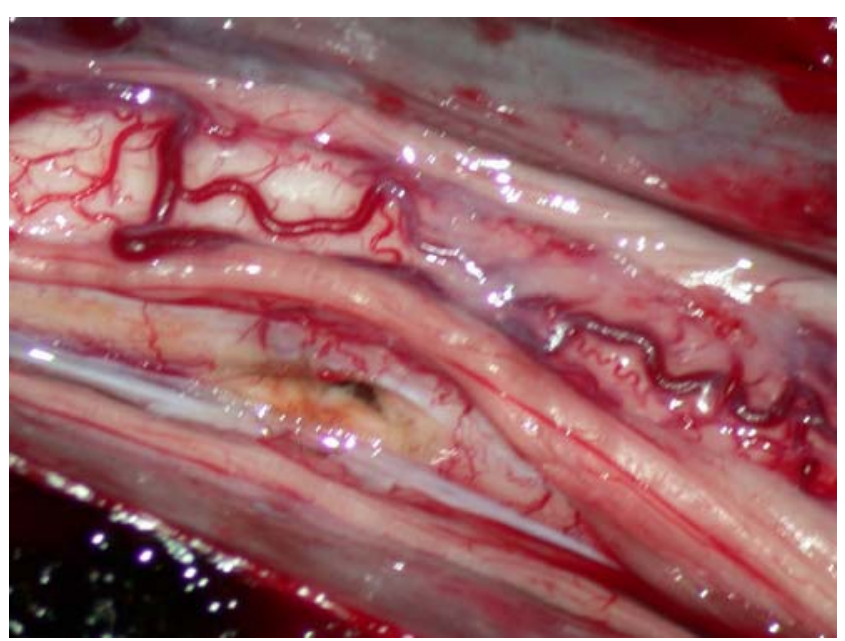

Fig. 4 Intraoperative photograph showing the plane of hemosiderin stained gliosis preserved after resection of the cavernoma

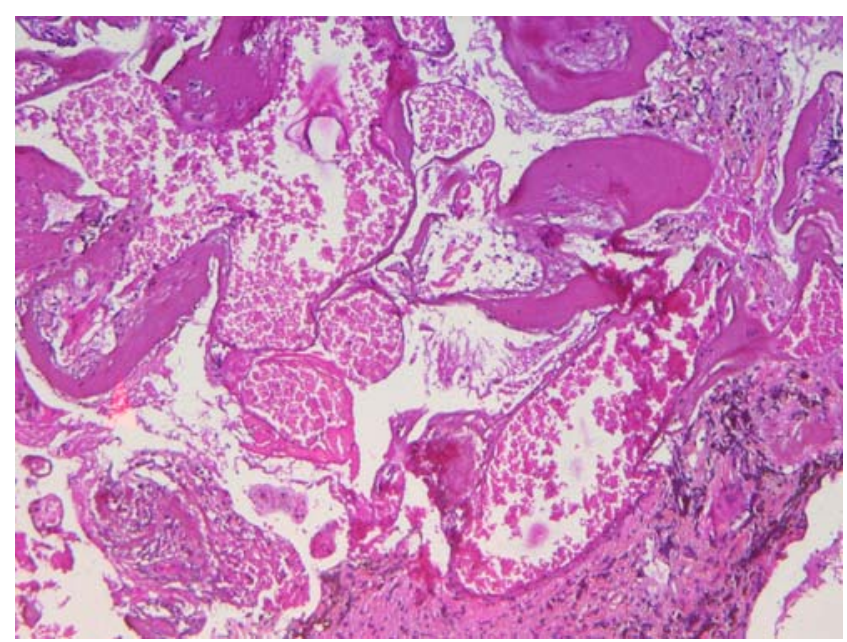

Fig. 5 Photomicrograph showing histopathological features of cavernous malformation: capillary vessels and veins with thick walls. Hematein phloxin safran. Original magnification, $40 \times$

lesion, predominantly with low intensity within the spinal cord. Smaller areas of increased signal intensity within or around the lesion may be present, and a rim of low-signal intensity because of the hemosiderin pigment may surround the mass $[8,17]$.

Special features of ImCA in children

Intramedullary cavernous malformations of the spinal cord are very uncommon, especially in children where only ten cases have yet been reported, mostly as isolated cases [1, 3, 6, 14, 17]. In 2002, Nagib and O'Fallon [14] summarized nine cases including their two personal cases, and in 2006, Bakir et al. [3] reported another pediatric patient with ImCA: Although the number of reported pediatric cases with ImCA is small, we can outline some differences from that of the

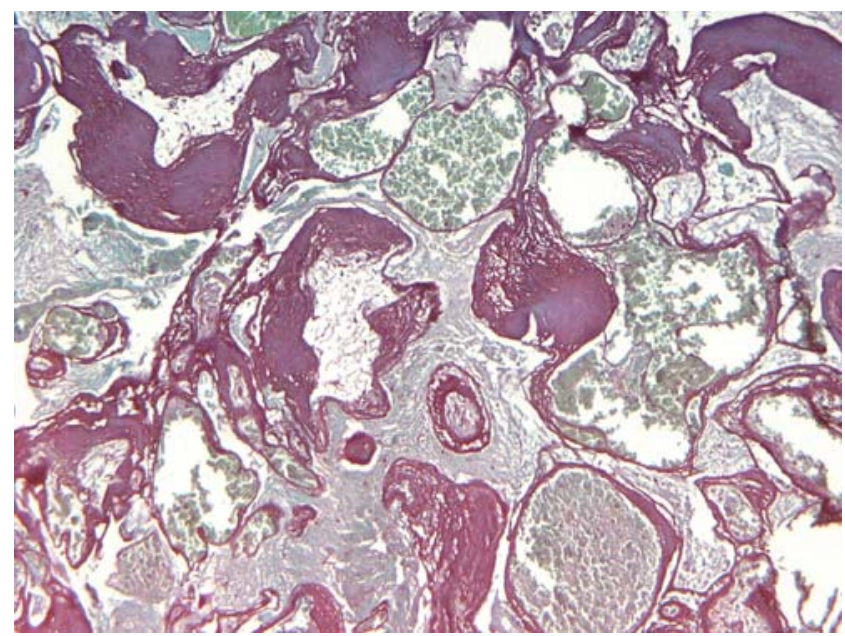

Fig. 6 Photomicrograph showing histopathological features of cavernous malformation: elastic coloration revealed the absence of internal elastic laminae. Orcein. Original magnification, 40× 


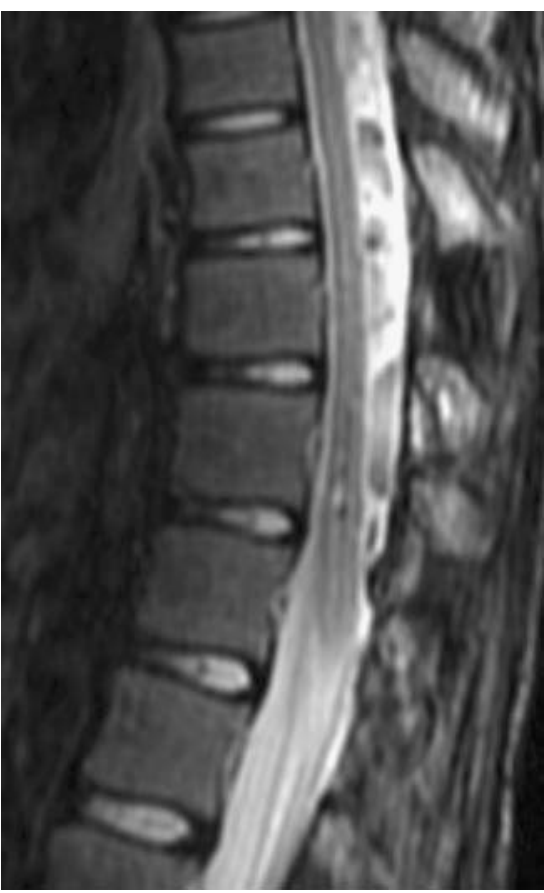

Fig. 7 Postoperative MRI study: sagittal T2-weighted images showing a slit postoperative cavity of mixed signal

adult regarding their epidemiological features and clinical presentation.

While ImCA in the adult occur more frequently in women than in men $[12,17]$, with a female/male ratio of approximately $2: 1$, seven of ten cases of symptomatic ImCA reported in children were male, thus accounting for a male/ female ratio of 2:1. The hypothesis of a hormonal effect leading to a greater risk of bleeding in female adults does not seem to be verified in children, but the mean age at bleeding is 13.2 years (range 8-17) and can also suggest the hormonal influence of puberty.

Moreover, although in adults, cavernous malformations occur mostly in the thoracic spinal cord, in children, ImCA appear to be evenly distributed in the thoracic and cervical spinal cord.

The common clinical presentation of ImCA in adults is a slow and progressive myelopathy $[1,4,11]$ or an acute deficit followed by a slow, stepwise deterioration with periods of clinical improvement because of repeated microhemorrhages followed by gliosis or iterative thrombosis within the cavernoma, resulting in altered blood flow in the adjacent neural parenchyma $[7,14]$. Contrary to these classical forms of presentation, all reported symptomatic children with ImCA presented with acute and severe neurological deficit followed by a rapid decline $[1,3,4,6,14,17]$ ascribed to an acute hemorrhage within the spinal cord parenchyma [7]. In our patient, the clinical presentation followed that pattern with acute neurological deficit because of hematomyelia documented on MRI.
Differences between intracerebral and intraspinal CA

A higher risk of bleeding has been identified for ImCA: The actuarial risk of bleeding of cerebral sustentorial lesions is $0.2-0.7 \%$ per year $[5,13,16]$ against $1.6 \%$ per year in $\operatorname{ImCA}[11,14]$ and $2.7 \%$ for brainstem CAs [10]. Moreover, the potential for a devastating hemorrhage with significant morbidity is higher for ImCA than for their cerebral counterpart because of the exiguous parenchymatous space where these lesions are located. As a result, surgical resection is strongly advocated in ImCA $[1,3,11,14]$.

\section{Surgical treatment}

Therapeutic indications remain controversial for asymptomatic cavernous malformations, except for accessible ImCA, which are recommended to be surgically removed [14]. Deeper, asymptomatic lesions are more likely managed expectantly, with a regular clinicoradiological follow-up [1, $7,12,14,19]$. In addition, asymptomatic ImCA are often diagnosed during the workup for systemic cavernomatosis, and systematic removal of asymptomatic $\mathrm{CA}$ in these patients is debatable.

When indicated, surgical resection can be achieved safely using microsurgical techniques [1, 19]: Dissection is started either directly over the lesion when a typical bluish discoloration is evident through the surface of the spinal cord or via a midline myelotomy for deeper lesions. Dissection must be confined to the thin plane of hemosiderin-laden gliosis surrounding the malformation to limit damage to the normal adjacent spinal cord tissue, thus making unnecessary the use of neurophysiologic monitoring that may be time-consuming and of debated reliability. Anson and Spetzler [1] have recommended removal of this

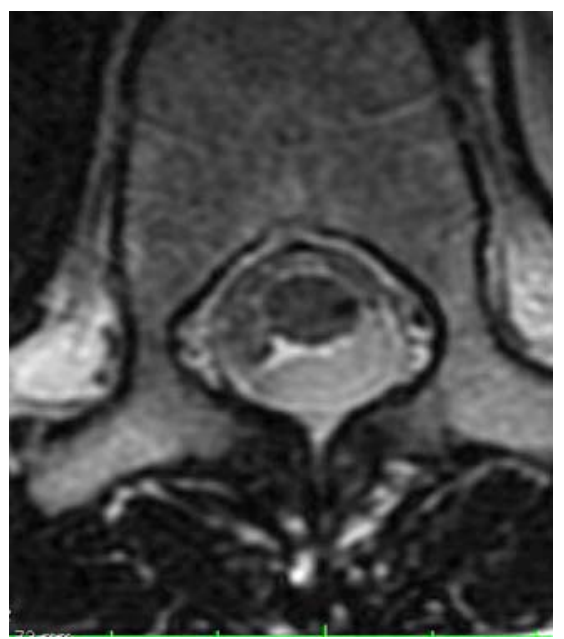

Fig. 8 Axial T2-weighted image revealing a small ovoid hyposignal beneath the surface of the left lateral column of the spinal cord because of the hemosiserin-laden tissue 
perilesional tissue to ensure total resection and prevent recurrence from this granulation formation, but we think, like others [19], that it must be preserved.

\section{Postoperative outcome}

The immediate postoperative course can be marked by transient neurological worsening [19] with good outcome at long-term follow-up [14], as in the present case.

In cases reported in adults, delayed complications resulted mostly from incomplete resection with recurrent bleeding $[1,19]$ requiring a "second-look" surgery for the residual cavernous malformation.

In children, another potential adverse effect of surgery is the risk of spinal deformity after laminectomy because of spinal growth: Among nine pediatric cases collected by Nagib and O'Fallon [14], no postoperative spinal deformity occurred among seven cases of laminectomy or laminoplasty, neither in our patient after a three-level laminotomy and laminoplasty. It remains to be proven that replacement laminoplasty techniques can prevent postoperative spinal deformities; however, these are recommended to preserve the anatomical planes and facilitate greatly subsequent surgery [14], including spinal fusion when needed.

The interpretation of postoperative MRI findings could be delicate because the low-intensity signal of postoperative scar tissue can be misdiagnosed for a residual cavernous malformation: It is more reliable 6 months later. MRI of the entire neuraxis is recommended to check for possible multiple lesions because ImCA are often associated with multiple intracranial cavernous malformations or with a familial disease caused by a mutation of $\mathrm{CH} 1$ or $\mathrm{CH} 2[1,14,19]$.

\section{Conclusion}

The epidemiological and clinical features of ImCA in children are different from adults: The sex ratio is male-biased, the distribution is equal between the thoracic and cervical spinal cord, and the clinical presentation is acute and severe.

Because ImCA have a higher risk of bleeding with catastrophic clinical consequences compared to cerebral CA, the risks of surgery need to be weighted against the risk of spontaneous deterioration on a case-by-case basis.

Microsurgical resection is the only treatment and has proved to be safe when operative guidelines are followed.

\section{References}

1. Anson JA, Spetzler RF (1993) Surgical resection of intramedullary spinal cord cavernous malformation. J Neurosurg 78:446451

2. Awad IA, Robinson JR Jr, Mohanty S, Estes ML (1993) Mixed vascular malformations of the brain: clinical and pathogenetic considerations. Neurosurgery 33:179-188

3. Bakir A, Savas A, Yilmaz E, Svas B, Erden E, Caglar S et al (2006) Spinal intradural-intramedullary cavernous malformation: case report and literature review. Pediatr Neurosurg 42:35-37

4. Cosgrove GR, Bertrand G, Fontaine S, Robitaille Y, Melanson D (1988) Cavernous angiomas of the spinal cord. J Neurosurg 68:31-36

5. Del Curling O Jr, Kelly DL Jr, Elster AD, Craven TE (1991) An analysis of a natural history of cavernous angiomas. J Neurosurg 75:702-708

6. Deutsch H, Jallo G, Faktorovitch A, Epstein F (2000) Spinal intramedullary cavernoma: clinical presentation and surgical outcome. J Neurosurg 93(1 Suppl):65-70

7. Fazi S, Menei P, Mercier P, Dubas F, Guy G (1992) Cavernomas of the spinal cord: report of two patients. Br J Neurosurg 6:149 152

8. Fontaine S, Melanson D, Cosgrove R, Bertrand G (1988) Cavernous hemangiomas of the spinal cord: MR imaging. Radiology 166:839-841

9. Fox MW, Onofrio BM (1993) The natural history and management of symptomatic and asymptomatic vertebral hemangiomas. J Neurosurg 78:36-45

10. Fritschi JA, Reulen HJ, Spetzler RF, Zabramski JM (1994) Cavernous malformations of the brainstem. A review of 139 cases. Acta Neurochir (Wien) 130:35-46

11. Ghogawala Z, Ogilvy CS (1999) Intramedullary cavernous malformations of the spinal cord. Neurosurg Clin N Am 10: 101-111

12. Harrison MJ, Eisenberg MB, Ullman JS, Oppenheim JS, Camins MB, Post KD (1995) Symptomatic cavernous malformations affecting the spine and spinal cord. Neurosurgery 37:195-205

13. Kondziolka D, Lunsford LD, Kestle JR (1995) The natural history of cerebral cavernous malformations. J Neurosurg 83:820-824

14. Nagib MG, O'Fallon MT (2002) Intramedullary cavernous angiomas of the spinal cord in the pediatric age group: a pediatric series. Pediatr Neurosurg 36:57-63

15. Ogilvy CS, Louis DN, Ojemann RG (1992) Intramedullary cavernous angiomas of the spinal cord: clinical presentation, pathological features, and surgical management. Neurosurgery 31:219-230

16. Robinson JR, Awad IA, Little JR (1991) Natural history of the cavernous angioma. J Neurosurg 75:709-715

17. Scott RM, Barnes P, Kupsky W, Adelman LS (1992) Cavernous angiomas of the central nervous system. J Neurosurg 76:38-46

18. Simard JM, Garcia-Bengochea F, Ballinger WE Jr, Mickle JP, Quisling RG (1986) Cavernous angiomas: a review of 126 collected and 12 new clinical cases. Neurosurgery 18:162-172

19. Vishteh AG, Zabramski JM, Spetzler RF (1999) Patients with spinal cord cavernous malformations are at increased risk for multiple neuraxis cavernous malformations. Neurosurgery 45:30-33 